\title{
EVALUACIÓN Y DIAGNÓSTICO DE ELEMENTOS DE CONCRETO LOCALIZADOS EN AMBIENTE MARINO DEL DISTRITO DE ITE, TACNA
}

\author{
EVALUATION AND DIAGNOSTIC OF CONCRETE ELEMENTS LOCALIZED \\ IN MARINO ENVIRONMENT OF THE ITE DISTRICT, TACNA
}

Milagros Natali Laguna Chite ${ }^{1}$ Alonso Dorian Mamani Antipuerta ${ }^{2}$

Cesar Cruz Espinoza ${ }^{3}$

Información del artículo: Recibido: 05/08/2020

Aceptado: 15/11/2020

1,2 Escuela de Ingeniería Civil, Universidad Privada de Tacna, Perú ${ }^{3}$ Docente Ingeniería civil, Universidad Privada de Tacna, Perú E-mail: ${ }^{1}$ milagros.laguna65@gmail.com, ${ }^{2}$ dorian_A95@hotmail.com, ${ }^{3}$ cruz_cesar5@hotmail.com 


\section{Resumen}

En la región de Tacna, las edificaciones con cercanía al mar frecuentemente presentan patologías en los elementos de concreto. Estas patologías con el tiempo generan el desarrollo del deterioro del mismo concreto afectando la durabilidad y el tiempo de vida útil de las edificaciones. La investigación tiene la finalidad de aportar conocimientos técnicos en las etapas de inicio, planificación y ejecución de edificaciones considerando la vulnerabilidad vinculada a un ambiente marino; mediante la evaluación de las propiedades mecánicas, físicas y químicas del concreto, diagnóstico de patologías y concluyendo con una propuesta de reparación. El lugar de investigación corresponde al Complejo Recreacional Municipal "Los Humedales de Ite", considerando que está expuesto a un ambiente marino y a una distancia al mar menor de $2 \mathrm{~km}$. Para el desarrollo de la investigación se empleó la inspección preliminar y detallada. La inspección preliminar identificó las condiciones de exposición (características meteorológicas), y la inspección detallada determinó las propiedades del concreto mediante los ensayos de laboratorio. Los resultados se evaluaron considerando los estándares de las normas ACI, NTP, ASTM y ASOCRETO; concluyéndose que la calidad del concreto es deficiente para una atmósfera marina severa debido a que el transporte de agentes nocivos se da través de la porosidad y absorción del concreto. Las características fisicoquímicas de los materiales indicaron que el ingrediente contaminado es el agregado fino debido a su alto índice de cloruros, factor que suma el proceso de corrosión del acero de refuerzo.

Palabras claves: Patología; concreto; ambiente marino.

\section{Abstract}

In the Tacna region, buildings close to the sea often present pathologies in the concrete elements. These pathologies over time generate the development of the deterioration of the same concrete affecting the durability and service life of the buildings. The research aims to provide technical expertise in the stages of the state, planning and execution of buildings considering the vulnerability linked to a marine environment; by evaluating the mechanical, physical and chemical properties of concrete, diagnosing pathologies and concluding with a repair proposal. The research site corresponds to the Municipal Recreation Complex "Los Humedales de Ite", considering that it is exposed to a marine environment and a distance to the sea less than $2 \mathrm{~km}$. Preliminary and detailed inspection was used for the development of research. Preliminary inspection identified exposure conditions (weather characteristics), and detailed inspection determined concrete properties through laboratory testing. The results were evaluated by considering the standards of the ACI, NTP, ASTM and ASOCRETO standards; it is concluded that the quality of the concrete is poor for a severe marine atmosphere because the transport of harmful agents occurs through the porosity and absorption of the concrete. The physicochemical characteristics of the materials indicated that the contaminated ingredient is the fine aggregate due to its high chloride index, a factor that adds up to the corrosion process of the reinforcing steel.

Keywords: Pathology; concrete; marine environment. 


\section{Introducción}

La escasa aplicación de procesos constructivos, conocimiento técnico, control de calidad y diseños estructurales adecuados, han afectado la durabilidad y tiempo de vida útil de las edificaciones cercanas al mar. Su cercanía al mar y la agresividad del medio ambiente, hacen que el concreto sea propenso al ataque de agentes nocivos como: cloruros, sulfatos y carbonatación. Estos agentes nocivos al estar en contacto con el concreto generan la presencia de patologías. El estudio del deterioro del concreto es un tema muy particular debido a los diferentes tipos por origen: físico, químico, fisicoquímico y estructural. Sin embargo, el deterioro que demanda mayor costo y complicaciones en la reparación, es el deterioro por origen químico. Este tipo de deterioro, se manifiesta fundamentalmente por la presencia de agua en el concreto proveniente de la humedad relativa y brisa marina propia de un ambiente marino (Pancorbo, 2013). Asimismo, las condiciones para que inicie el ataque químico es el transporte de agentes nocivos a través de los poros del concreto, de forma que si no hay transporte no hay reacción y por lo tanto no se genera la corrosión del acero de refuerzo (Porto, 2005).

Por estas razones, se requiere un adecuado diseño y control de calidad de las edificaciones en ambiente marino considerando no solo la resistencia como un indicador de la calidad del concreto, sino también la vulnerabilidad vinculada a la zona de exposición.

\section{Objetivo}

Diagnosticar el origen de las patologías de los elementos de concreto, a través de la evaluación de las condiciones de exposición (características meteorológicas), y las actuales propiedades mecánicas, físicas y químicas de los elementos de concreto localizados en ambiente marino del distrito de Ite, región Tacna

\section{Metodología}

En la investigación desarrolló el diagnóstico de elementos de concreto utilizando los métodos de inspección preliminar, inspección detallada y evaluación, con la finalidad de utilizar procedimientos estandarizados y aceptados por una comunidad científica, para el caso, la aplicación de las normativas del Instituto Americano del Concreto (ACl), Norma Técnica Peruana (NTP), Asociación Americana de Ensayo de Materiales (ASTM), Asociación Colombiana de Productores de Concreto (ASOCRETO).

\section{Inspección preliminar}

En la Inspección preliminar se dictaminó la posible causa y factores concurrentes de las patologías de los elementos de concreto en base de parámetros normativos de la "Guía para la evaluación de estructuras de hormigón antes de la rehabilitación - $\mathrm{ACl} 364$ ", donde se clasifica la 
información en función de antecedentes y estudios previos, características de agentes nocivos en contacto con la estructura, características meteorológicas y naturaleza de los materiales.

La primera fuente de información corresponde al Expediente Técnico del Complejo Recreacional Municipal "Los Humedales de Ite", donde se señalan los datos de tiempo en servicio de la estructura, mantenimientos y reparaciones anteriores, la naturaleza y procedencia de los materiales para la elaboración del concreto y características técnicas conforme a los elementos de concreto. Estos datos fueron obtenidos de documentos existentes de la Municipalidad Distrital de Ite (MDI).

La segunda fuente de información corresponde a las características meteorológicas, donde se señalan los datos como la temperatura $\left({ }^{\circ} \mathrm{C}\right)$, humedad relativa $(\%)$ y precipitación $(\mathrm{mm})$ que son indicadores de la agresividad del medio ambiente. Estos datos fueron obtenidos de la Estación Meteorológica Convencional del Distrito de Ite mediante el Servicio Nacional de Meteorología e Hidrología del Perú - SENAMHI. En la Figura 1, se observa la localización del Complejo Recreacional en ambiente marino propio de la cercanía al mar.

\section{Figura 1 \\ Localización del Complejo Recreacional Municipal "Los Humedales de Ite" en ambiente marino en el Distrito de Ite.}

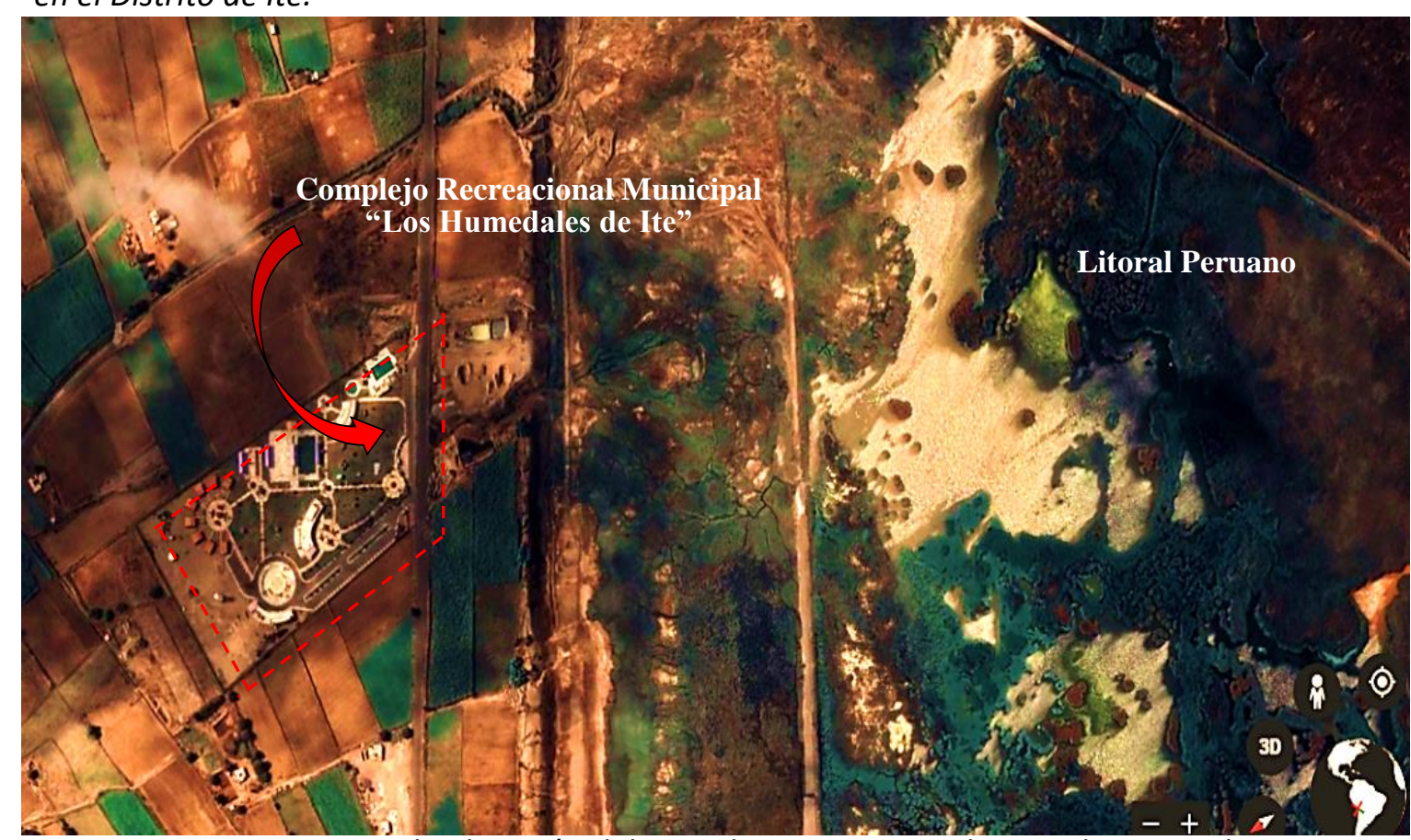

Nota. La Figura representa la ubicación del Complejo Recreacional a una distancia al mar menor de 2 km. Fuente. Google Earth.

La tercera fuente de información corresponde a los agentes nocivos en contacto de la estructura, donde se señala los datos como la atmosfera, agua, suelo, brisa marina y niebla marina que serán indicadores de las posibles causas de las patologías. Estos datos fueron obtenidos de documentos existentes respaldados por profesionales de la Municipalidad Distrital de Ite. 


\section{Inspección Detallada}

La Inspección detallada cuantifica la extensión del deterioro y caracteriza todos los elementos de concreto con patologías, su desarrollo exige la elaboración de ensayos que certifiquen la calidad de los resultados en base a las Norma Técnica Peruana (NTP).

La primera disponibilidad fue la elaboración de los ensayos de laboratorio para determinar y evaluar las actuales propiedades mecánicas, químicas y físicas del concreto. En la Tabla 1, se observa los ensayos que se realizaron para evaluar la calidad del concreto.

Tabla 1

Ensayos realizados para evaluación de la calidad del concreto

\begin{tabular}{llll}
\hline \multicolumn{1}{c}{ Ensayos in situ } & \multicolumn{3}{c}{ Ensayos de laboratorio } \\
\cline { 2 - 4 } & \multicolumn{1}{c}{ Mecánico } & \multicolumn{1}{c}{ Físico } & \multicolumn{1}{c}{ Químico } \\
\hline Extracción de diamantina & Resistencia a & Densidad, & Determinación de \\
(NTP 339.059 & Compresión - & Absorción y & Cloruros (NTP 339.177) \\
Resistencia a Compresión & Testigos de & Porosidad & Determinación de \\
Mediante Esclerómetro & Concreto (NTP & (NTP 339.187) & Sulfatos (NTP 339.178) \\
(NTP 339.181) & $339.034)$ & & Determinación de pH \\
& & & (NTC 5264) \\
Profundidad de & & Determinación de \\
Carbonatación (RILEM CPC- & & Carbonatación \\
18) & & & (Difracción de Rayos X) \\
\hline
\end{tabular}

Nota. Los ensayos fueron certificados por profesionales y responsables de los laboratorios.

Fuente. Tabla elaborada por los autores.

La segunda disponibilidad fue la elaboración de los ensayos de laboratorio correspondiente a los materiales utilizados en la elaboración del concreto (agua, agregado fino y agregado grueso). En la Tabla 2, se observa los ensayos que se realizaron para descartar la causa de materiales contaminantes.

\section{Tabla 2}

Ensayos realizados para la evaluación de los materiales utilizados en la elaboración del concreto

\begin{tabular}{lll}
\hline \multicolumn{1}{c}{ Agregado grueso } & \multicolumn{1}{c}{ Agregado fino } & \multicolumn{1}{c}{ Agua para el concreto } \\
\hline Determinación de Cloruros & Determinación de & Determinación de $\mathrm{pH}$ \\
(NTP 339.177) & Cloruros (NTP 339.177) & (NTP 214.029) \\
Determinación de Sulfatos & Determinación de & Determinación de \\
(NTP 339.178) & Sulfatos (NTP 339.178) & cloruros (NTP 339.076) \\
& & Determinación de \\
& & sulfatos (NTP 339.074) \\
\hline
\end{tabular}

Nota. Los ensayos fueron certificados por profesionales y responsables de los laboratorios. Fuente. Tabla elaborada por los autores. 


\section{Evaluación de patologías}

La Evaluación de patologías describe el tipo de deterioro y posible causal de las patologías en base a sustento técnico y bibliográfico, con el objetivo de proponer reparación.

La primera disposición fue el levantamiento de patologías mediante la elaboración de planos, donde se localizan y dimensionan las patologías según registro fotográfico.

La segunda disposición fue la selección de elementos con patologías, donde se identificando el porcentaje (\%) de elementos dañados por cada ambiente del Complejo Recreacional.

La tercera disponibilidad fue la evaluación de niveles de severidad, tanto a nivel de patologías como de elementos de concreto, considerando los criterios como: el tipo de patología, su espesor, área afectada; valores numéricos expresados en $\mathrm{m} 2$ y también por consiguiente en valores porcentuales. En la Tabla 3 y 4 , se observa los indicadores para definir el nivel de severidad de las patologías y elementos de concreto respectivamente.

La cuarta disponibilidad fue el examen general de patologías en relación de las características patológicas considerando la localización y dimensión de patologías; y fundamentos teóricos de acuerdo al deterioro del concreto por daños de origen físico, químico, fisicoquímico y estructural.

\section{Tabla 3}

Indicadores para definir el nivel de severidad de las patologías

\begin{tabular}{|c|c|c|}
\hline Patología & Nivel de severidad & Descripción \\
\hline \multirow{3}{*}{ Fisuras (mm) } & Leve & Abertura de $0.05 \mathrm{~mm}$ a 0,30 \\
\hline & Moderado & Abertura de $0.30 \mathrm{~mm}$ a 0,50 \\
\hline & Severo & Abertura de $0.50 \mathrm{~mm}$ a 1,00 \\
\hline \multirow{3}{*}{ Grietas $(\mathrm{mm})$} & Leve & Abertura de $1,00 \mathrm{~mm}$ a 3 \\
\hline & Moderado & Abertura de $3 \mathrm{~mm}$ a 5 \\
\hline & Severo & Abertura de 5 mm a 10 \\
\hline \multirow{3}{*}{ Humedad (\%) } & Leve & Porcentaje no mayor a 25 \\
\hline & Moderado & Porcentaje no mayor a 35 \\
\hline & Severo & Porcentaje no mayor a 50 \\
\hline \multirow{3}{*}{ Eflorescencia (\%) } & Leve & Porcentaje no mayor a 25 \\
\hline & Moderado & Porcentaje no mayor a 35 \\
\hline & Severo & Porcentaje no mayor a 50 \\
\hline \multirow{3}{*}{$\begin{array}{c}\text { Descascaramiento } \\
\qquad(\%)\end{array}$} & Leve & Porcentaje no mayor a 25 \\
\hline & Moderado & Porcentaje no mayor a 35 \\
\hline & Severo & Porcentaje no mayor a 50 \\
\hline
\end{tabular}

Nota. Los valores de la siguiente tabla están en base a otras investigaciones Fuente. Tabla elaborada por los autores. 


\section{Tabla 4}

Indicadores para definir el nivel de severidad en elementos de concreto

\begin{tabular}{cc}
\hline Nivel & Parámetro \\
\hline Leve & $\mathrm{X}<15 \%$ del elemento \\
Moderado & $15 \%<\mathrm{X}<40 \%$ del elemento \\
Severo & $\mathrm{X}>40 \%$ del elemento
\end{tabular}

Nota. Los valores de la siguiente tabla están en base a otras investigaciones.

Fuente. Tabla elaborada por los autores.

En la Figura 2, se observa el proceso de la metodología para el desarrollo del diagnóstico de patologías

Figura 2

Proceso para el desarrollo del diagnóstico de patologías

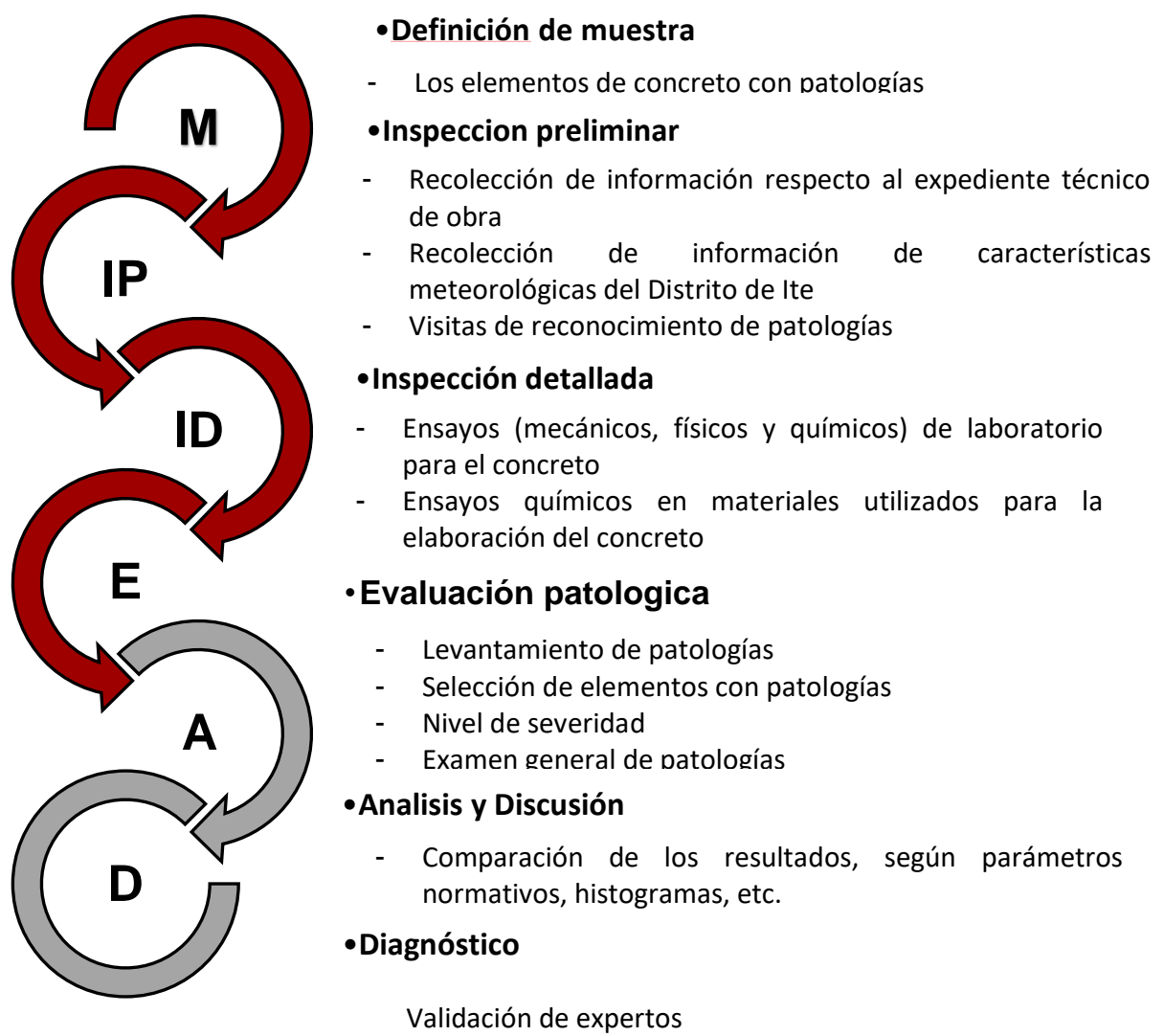

Nota. Los ensayos fueron certificados por profesionales y responsables de los laboratorios. Fuente. Figura elaborada por los autores. 


\section{Resultados}

\section{Inspección Preliminar}

En la Figura 3, se observan los resultados de las fichas de información general que proporcionan los antecedentes de intervenciones y/o mantenimientos con respecto al Complejo Recreacional respectivamente.

\section{Figura 3}

Antecedentes del Complejo Recreacional

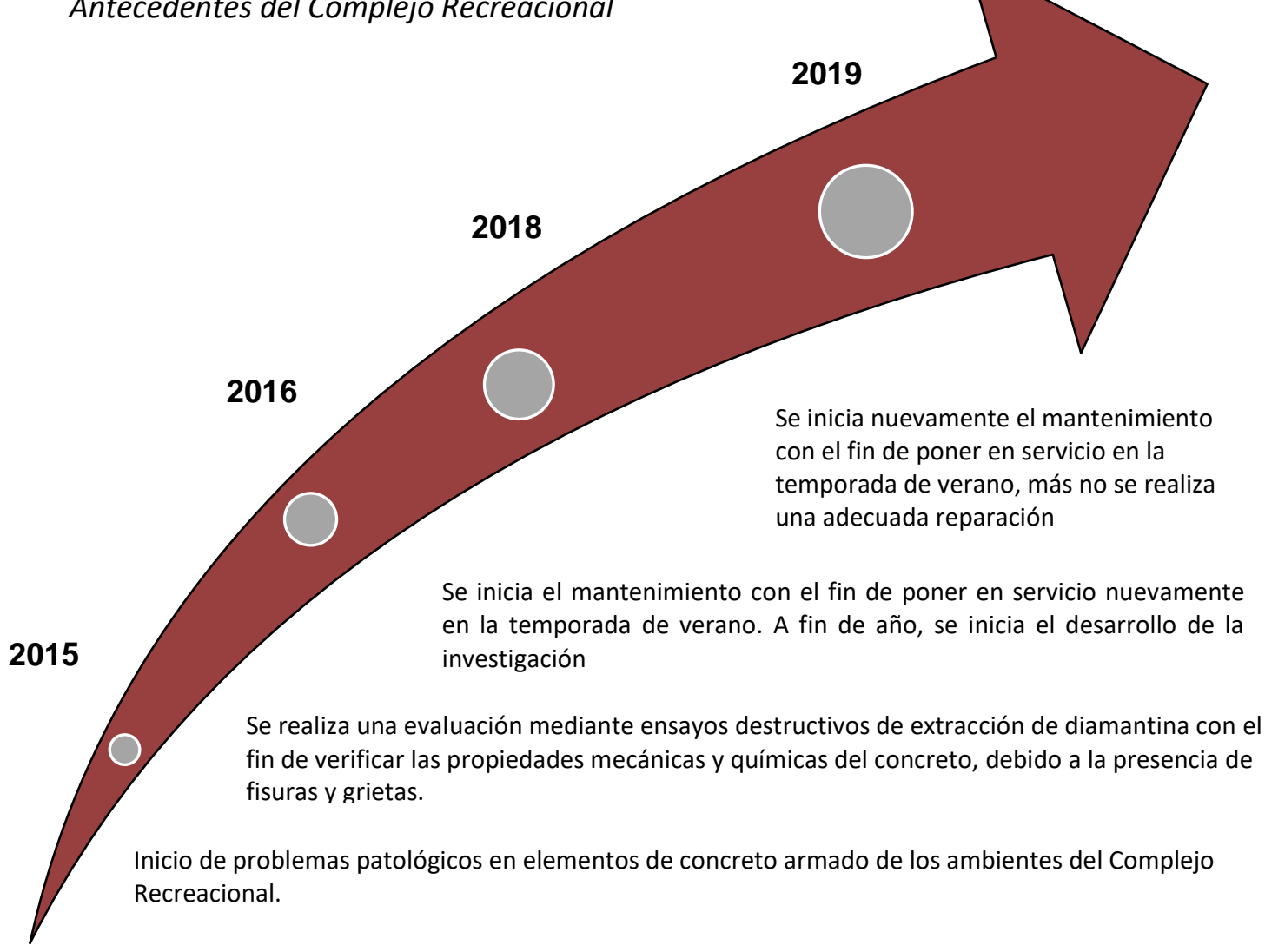

Nota. Los datos fueron obtenidos de los documentos respaldados por profesionales de la Municipalidad Distrital de Ite. Fuente. La Figura fue elaborada por los autores.

En la Tabla 5 y 6, se observan los resultados de la ficha de características meteorológicas del distrito de Ite y las características de agentes nocivos en contacto con la estructura respectivamente. 


\section{Inspección Detallada}

En la Tabla 7, se observa los resultados de los ensayos mecánicos, físicos y químicos en los elementos de concreto armado. Los ensayos de laboratorio se clasificaron en: ensayos mecánicos, donde se desarrolló la resistencia a comprensión mediante la extracción de diamantinas; ensayos físicos, donde se desarrolló la densidad, absorción y porosidad del concreto y ensayos químicos, donde se determinaron el contenido de cloruros, sulfatos, carbonatación mediante la difracción de rayos $\mathrm{X}$.

\section{Tabla 5}

Características meteorológicas del distrito de Ite

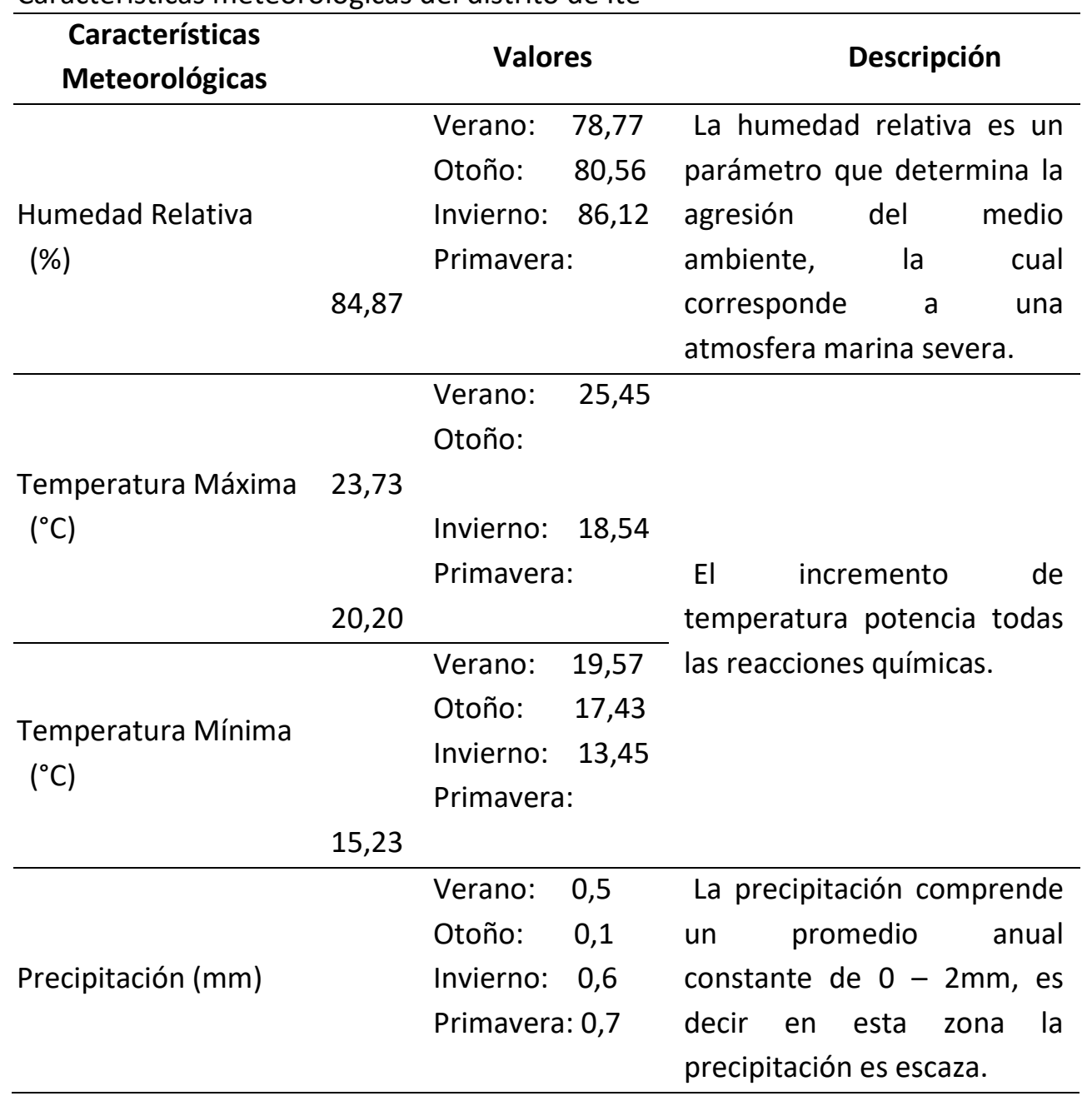

Nota. Los datos fueron obtenidos de los documentos respaldados por SENAMHI. Fuente. Tabla elaborada por los autores. 
Tabla 6

Características de agentes nocivos en contacto con la estructura

\begin{tabular}{|c|c|c|c|}
\hline & Agentes & Valores & Descripción \\
\hline ra (\%) & Atmosfe & HR : 82,50 & $\begin{array}{l}\text { La atmosfera es marina, } \\
\text { por la ubicación cercana al litoral } \\
\text { peruano con presencia de brisa, } \\
\text { niebla marina y características } \\
\text { climatológicas propias del lugar. }\end{array}$ \\
\hline (ppm) & Agua & $\begin{array}{l}\text { Cloruros: } 240 \\
\text { Sulfatos: } 600\end{array}$ & $\begin{array}{l}\text { El agua de riego es } \\
\text { proveniente del canal de } \\
\text { captación del rio Locumba. }\end{array}$ \\
\hline (ppm) & Suelo & $\begin{array}{l}\text { Según información } \\
\text { técnica, la composición } \\
\text { química del suelo es: } \\
\text { Sales solubles: } \\
7600 \text { Cloruros: } 900 \\
\text { Sulfatos: }\end{array}$ & \begin{tabular}{lrr}
\multicolumn{3}{c}{ Los suelos de la zona son } \\
de fines & agrícolas que \\
anualmente & reciben aguas \\
provenientes & de & las \\
precipitaciones atmosféricas y de \\
corrientes de agua superficial del \\
rio Locumba.
\end{tabular} \\
\hline Marina & $\begin{array}{l}\text { Brisa } \\
\text { (ppm) }\end{array}$ & $\begin{array}{l}\text { Según } \\
\text { fundamentos teórico, la } \\
\text { composición química del } \\
\text { mar peruano es: } \\
\text { Cloruros: } 23000 \\
\text { Sulfatos } 42750\end{array}$ & $\begin{array}{l}\text { La brisa marina } \\
\text { transporta } \\
\text { contenidos de humedad que, } \\
\text { lleva en sí cloruros y sulfatos; que } \\
\text { se depositan en la superficie del } \\
\text { concreto lo cual ocasiona causar } \\
\text { los daños. }\end{array}$ \\
\hline $\begin{array}{l}\text { Marina } \\
\text { ladera }\end{array}$ & $\begin{array}{l}\text { Neblina } \\
\text { y de } \\
(\mathrm{ppm})\end{array}$ & \begin{tabular}{lcc}
\multicolumn{3}{c}{ Según } \\
fundamentos & teórico, la \\
composición química & es \\
determinada & por & la \\
atmósfera & y & la \\
contaminación: & \\
Cloruros: 4,5 & \\
Sulfatos: $<5$ & \\
\end{tabular} & $\begin{array}{l}\text { Las nieblas costeras } \\
\text { generan una acción de roció lo } \\
\text { que produce la presencia de alta } \\
\text { de humedad y a la vez trasladan } \\
\text { agentes químicos según la } \\
\text { dirección del viento. }\end{array}$ \\
\hline
\end{tabular}

Nota. Los datos fueron obtenidos de los documentos respaldados por SENAMHI y la Municipalidad Distrital de Ite. Fuente. Tabla elaborada por los autores. 
Tabla 7

Ensayos mecánicos, físicos y químicos en elementos de concreto armado

\begin{tabular}{|c|c|c|c|c|c|c|c|}
\hline \multirow{3}{*}{$\begin{array}{l}\text { Ensayos de } \\
\text { Laboratorio }\end{array}$} & \multicolumn{7}{|c|}{ Elementos de Concreto } \\
\hline & \multicolumn{3}{|c|}{ Columnas } & \multicolumn{4}{|c|}{ Vigas } \\
\hline & C8-SR & C2-SC & C2-SUM & V1-SR & V3-SR & V4-SR & V3-SC \\
\hline $\begin{array}{l}\text { Resistencia a } \\
\text { Compresión NTP } \\
339.034 \\
(\mathrm{~kg} / \mathrm{cm} 2)\end{array}$ & 153,8 & 206,48 & - & 120,33 & 185,73 & - & 184,54 \\
\hline $\begin{array}{l}\text { Densidad NTP } 339.187 \\
(\mathrm{~kg} / \mathrm{cm} 2)\end{array}$ & - & - & 2629,25 & - & - & 2538,97 & - \\
\hline $\begin{array}{l}\text { Absorción NTP } 339.187 \\
\text { (\%) }\end{array}$ & - & - & 4,53 & - & - & 4,57 & - \\
\hline $\begin{array}{l}\text { Porosidad NTP } 339.187 \\
\text { (\%) }\end{array}$ & - & - & 6,96 & - & - & 7,23 & - \\
\hline PH (1:5) NTC5264 & 12,14 & - & - & 12,08 & - & - & 12,07 \\
\hline $\begin{array}{l}\text { Carbonatación Dif } \\
\text { Rayos X (\%) }\end{array}$ & 2,18 & - & - & 13,55 & - & - & 6,68 \\
\hline $\begin{array}{l}\text { Cloruros NTP } 339.177 \\
\text { (ppm) }\end{array}$ & 3645,91 & - & - & 4391,41 & - & - & 3759,15 \\
\hline $\begin{array}{l}\text { Sulfatos NTP } 339.178 \\
\text { (ppm) }\end{array}$ & 1088,50 & - & - & 985,55 & - & - & 1409,20 \\
\hline $\begin{array}{l}\text { Prof. Carbonatación } \\
\text { RILEM CPC-18 (mm) }\end{array}$ & 12,00 & 13,00 & 10,00 & 10,00 & - & - & 11,00 \\
\hline
\end{tabular}

Nota. Los datos fueron obtenidos de ensayos de laboratorio realizado en la Universidad Nacional de Ingeniería y la Universidad Privada de Tacna. Fuente. Tabla elaborada por los autores.

En la Figura 4, se observa la variación de las propiedades fisicoquímicas del concreto entre el periodo del 2016 - 2019 


\section{Figura 4}

Histogramas de variación de propiedades fisicoquímicas del concreto entre el periodo 2016 -2019
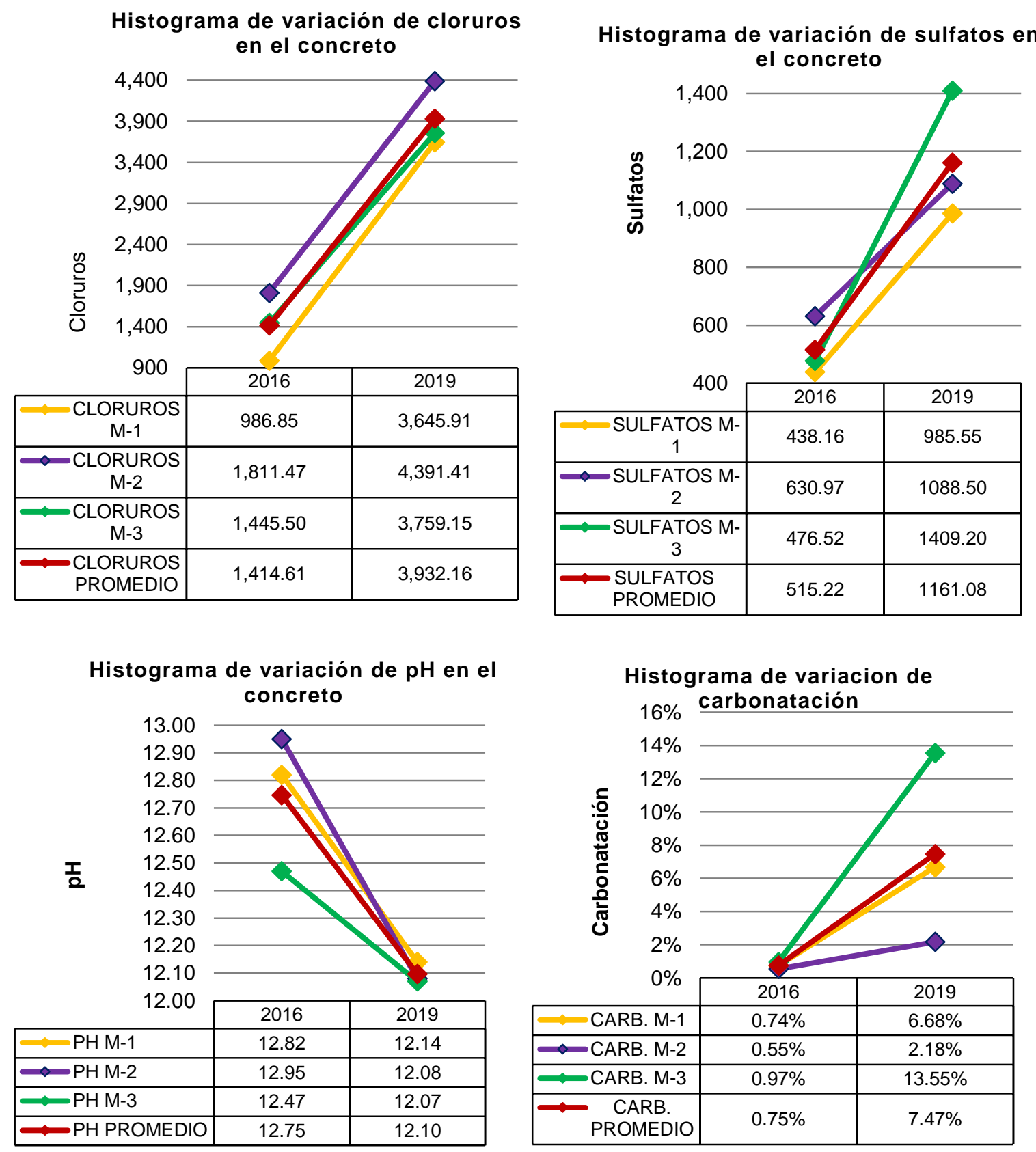

Nota. Los datos correspondientes al periodo 2016 son obtenidos de la Municipalidad Distrital de Ite abalado por profesionales y los datos correspondientes al periodo 2019 son obtenidos de los ensayos de laboratorio de la inspección detallada. Fuente. Figura elaborada por los autores.

En la Tabla 8, se observa los resultados de los ensayos químicos de los materiales para la elaboración del concreto (agregado fino, agregado grueso, agua). Los ensayos químicos fueron la determinación de cloruros, sulfatos y $\mathrm{pH}$. 


\section{Tabla 8}

Ensayos químicos de los materiales para la elaboración del concreto

\begin{tabular}{|c|c|c|c|c|c|}
\hline \multirow{2}{*}{$\begin{array}{l}\text { Ensayos de } \\
\text { laboratorio }\end{array}$} & \multirow{2}{*}{ Materiales } & \multirow{2}{*}{$\frac{\text { Columnas }}{\text { C-8 }}$} & \multicolumn{2}{|c|}{ Vigas } & \multirow{2}{*}{$\begin{array}{c}\text { Agua para el } \\
\text { concreto }\end{array}$} \\
\hline & & & V-1 & V-3 & \\
\hline Cloruros & Agr. Fino & 2755,67 & 3609,33 & 3087,25 & \\
\hline $\begin{array}{l}\text { NTP } 339.177 \\
\text { NTP } 339.076 \\
(p p m)\end{array}$ & Agr. Grueso & 650,24 & 542,08 & 431,90 & 240 \\
\hline Sulfatos & Agr. Fino & 332,25 & 256,32 & 678,65 & \\
\hline $\begin{array}{l}\text { NTP } 339.178 \\
\text { NTP } 339.074 \\
(p p m)\end{array}$ & Agr. Grueso & 156,25 & 129,23 & 130,55 & 600 \\
\hline $\mathrm{PH}(1: 5)$ & Canal de & - & - & & \\
\hline NTP 214.029 & $\begin{array}{c}\text { Captación de } \\
\text { Ite }\end{array}$ & & & & 8,71 \\
\hline
\end{tabular}

Nota. Los datos fueron obtenidos de ensayos de laboratorio realizado en la Universidad Nacional de Ingeniería. Fuente. Tabla elaborada por los autores.

\section{Evaluación de patologías}

El examen general de patologías desarrolla la evaluación en relación a la localización y dimensión de las patologías y fundamentos teóricos de acuerdo al deterioro del concreto para determinar el origen de las patologías en los elementos de concreto. En la Figura 5, se observa el examen general de patologías donde se describe el origen de los problemas patológicos predominantes. 


\section{Figura 5}

Examen general de patologías de los elementos de concreto

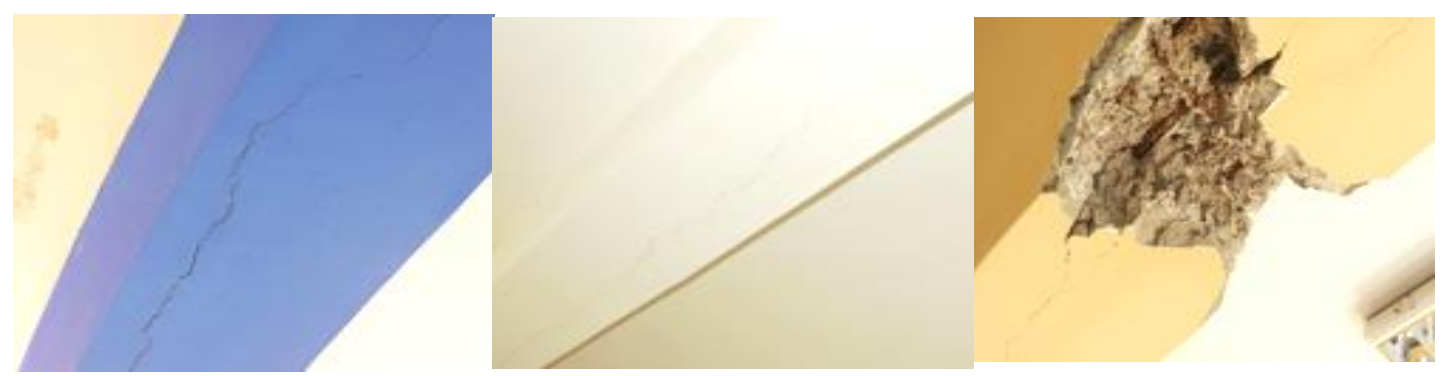

Fisuras y grietas por corrosión del acero de refuerzo

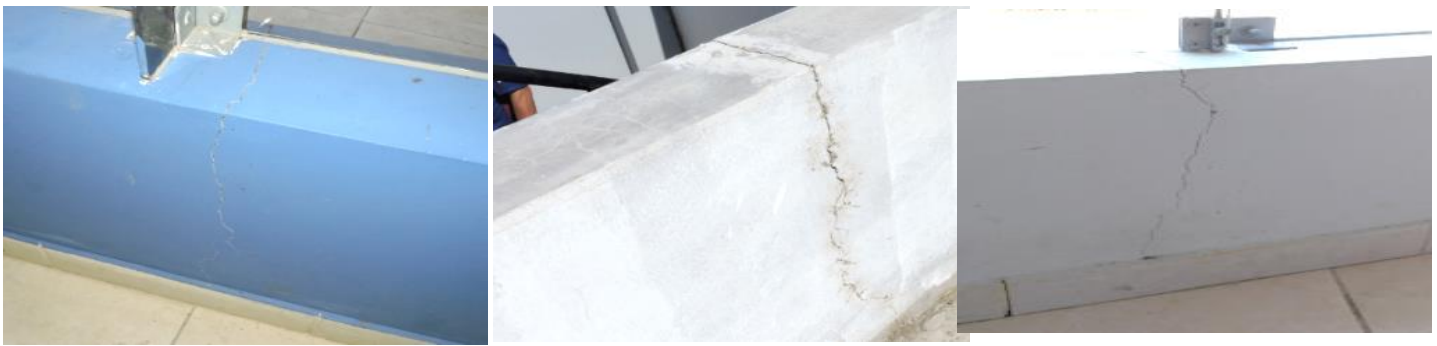

Fisuras y grietas por contracción térmica

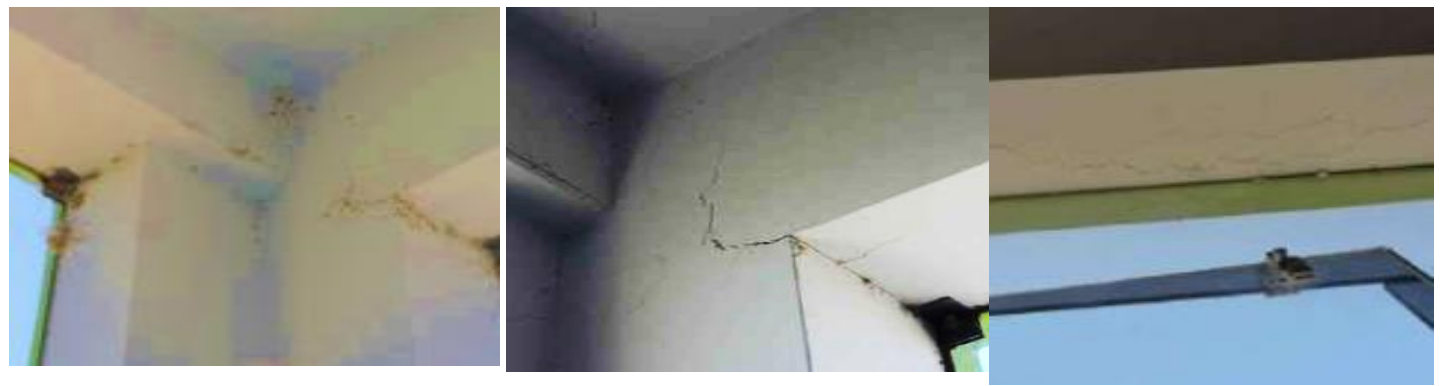

Fisuras por asentamiento de suelos expansivos

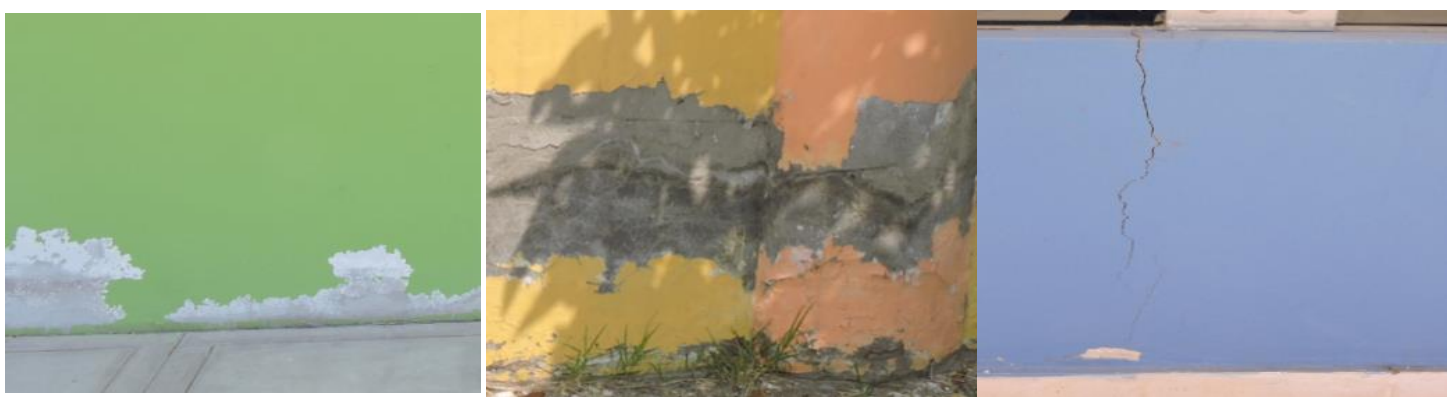

Fisuras y eflorescencia por acción de sulfatos

Nota. Las fotografías fueron obtenidas de las visitas de inspección. Fuente. Figura elaborada por los autores.

En la Tabla 9, se observa los resultados de la clasificación del nivel de severidad de los elementos de concreto que presentan patologías como fisuras, grietas, eflorescencia, humedad y desprendimientos. 


\section{Tabla 9}

Nivel de severidad de elementos de concreto con patologías

\begin{tabular}{|c|c|c|c|c|c|c|c|c|}
\hline \multirow{2}{*}{$\begin{array}{c}\text { Tipos de } \\
\text { elementos } \\
\text { de concreto }\end{array}$} & \multirow{2}{*}{$\begin{array}{c}\text { Elementos } \\
\text { de } \\
\text { concreto }\end{array}$} & \multirow{2}{*}{$\begin{array}{c}\text { Elementos } \\
\text { sin } \\
\text { patologías }\end{array}$} & \multicolumn{6}{|c|}{ Nivel de Severidad } \\
\hline & & & Leve & $\%$ & Moderado & $\%$ & Severo & $\%$ \\
\hline Vigas & 128 & 51 & 32 & 25,00 & 39 & 30,47 & 6 & 4,69 \\
\hline Columnas & 79 & 51 & 26 & 32,91 & 2 & 2,53 & 0 & 0,00 \\
\hline Placas & 21 & 11 & 8 & 38,10 & 2 & 9,52 & 0 & 0,00 \\
\hline Sobrecimiento & 66 & 36 & 10 & 15,15 & 20 & 30,30 & 0 & 0,00 \\
\hline Losas & 45 & 0 & 45 & 100,00 & 4 & 8,89 & 0 & 0,00 \\
\hline Escaleras & 4 & 0 & 1 & 0,82 & 6 & 4,92 & 0 & 0,00 \\
\hline Veredas & 149 & 51 & 54 & 44,26 & 37 & 30,33 & 7 & 5,74 \\
\hline Sardineles & 43 & 11 & 0 & 0,00 & 20 & 16,39 & 12 & 9,84 \\
\hline Total & 343 & 130 & 122 & 35,57 & 73 & 21,28 & 25 & 7,29 \\
\hline
\end{tabular}

Nota. Los datos fueron obtenidos de las visitas de inspección. Fuente. Tabla elaborada por los autores.

En la figura se hace una representación del nivel de severidad de los elementos de concreto con patologías.

\section{Figura 6}

Nivel de severidad de los elementos de concreto con patologías

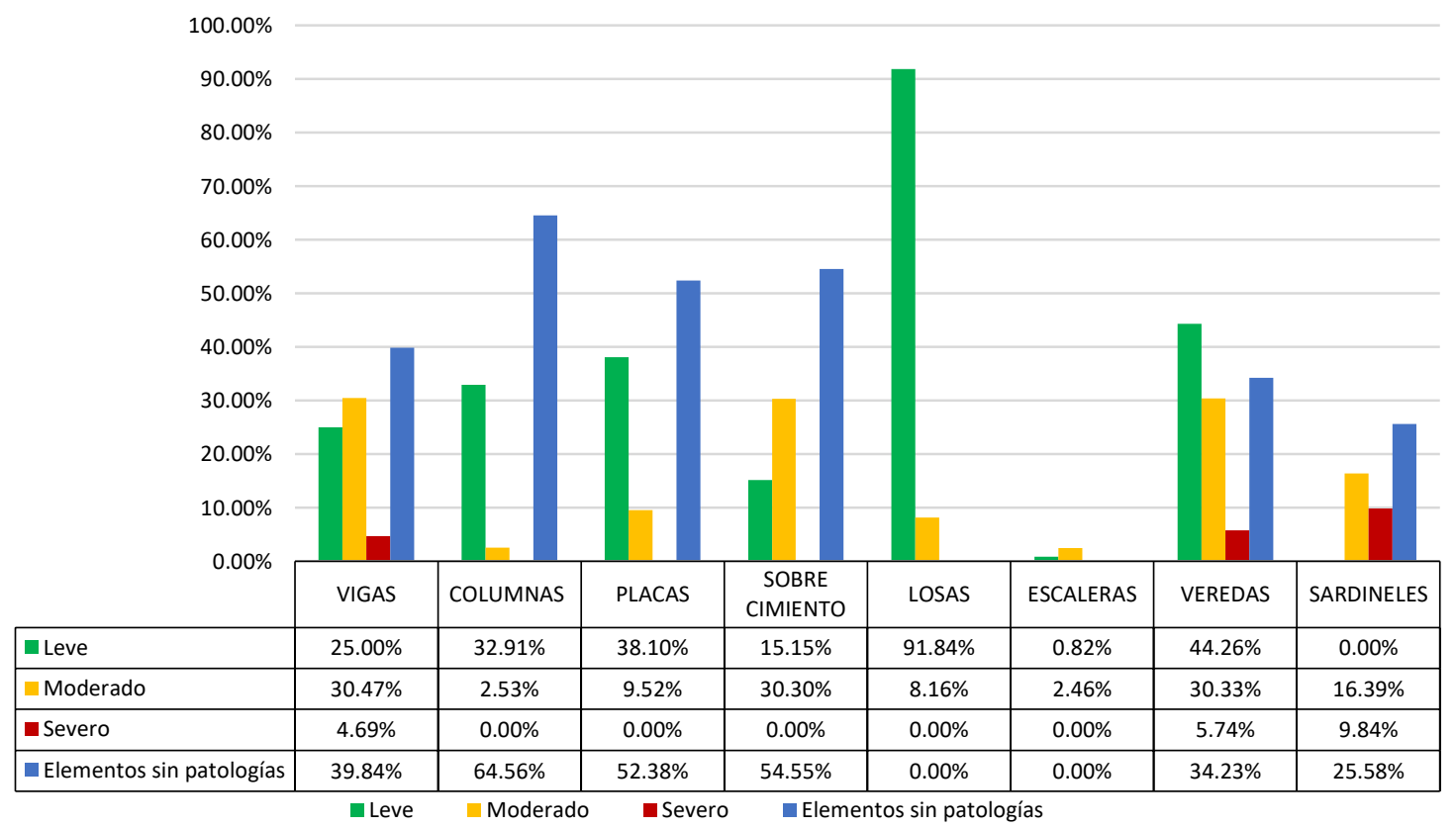

Nota. Los datos fueron obtenidos de las visitas de inspección. Fuente. Figura elaborada por los autores. 


\section{Discusiones}

La evaluación muestra los resultados de las condiciones de exposición, características del concreto, características de los materiales y nivel de severidad tras la aplicación de los métodos de inspección preliminar, inspección detallada y evaluación de patologías.

\section{Inspección Preliminar}

La agresividad del medio ambiente que rodea a los elementos de concreto del Complejo Recreacional fue determinada por las condiciones de exposición bajo las características meteorológicas del distrito de Ite (humedad relativa, temperatura y precipitación). La humedad relativa tiene un valor promedio anual de $82,50 \%$, que corresponde a una atmósfera marina severa. Generalmente el concreto es propenso a tener un rápido deterioro debido a que absorbe la humedad del medio ambiente y activa el mecanismo de daño por carbonatación a un nivel medio, un ataque químico a un nivel mínimo y una corrosión por efecto de carbonatación y cloruros a un nivel alto, mientras que la temperatura tiene un valor promedio anual de $25,45^{\circ} \mathrm{C}$ y $19,57{ }^{\circ} \mathrm{C}$ en verano, $23,73{ }^{\circ} \mathrm{C}$ y $17,43{ }^{\circ} \mathrm{C}$ en otoño, $18,44{ }^{\circ} \mathrm{C}$ y $17,43{ }^{\circ} \mathrm{C}$ en invierno; y $20,20{ }^{\circ} \mathrm{C}$ y $15,23{ }^{\circ} \mathrm{C}$ en primavera. El incremento de temperatura potencia todas las reacciones químicas, es decir, un aumento de temperatura en $10^{\circ} \mathrm{C}$ duplica la velocidad de la reacción química, lo cual hace que el deterioro se de en tiempos cortos (Sanchez de Guzman, 2004).

La precipitación tiene un valor promedio anual de $0,5 \mathrm{~mm}$; valor que afirma la presencia de lluvia ligera en la época de verano y lluvia intensa en la época de invierno y primavera. La presencia de ciclos de humedecimiento es índice esencial para el deterioro de los elementos de concreto. En la Figura 5, se observa los histogramas comparativos de las características meteorológicas del distrito de Ite en el periodo del $2012-2014$.

La agresividad del medio ambiente que rodea a los elementos de concreto del Complejo Recreacional fue determinada por los agentes nocivos en contacto con los elementos de concreto (atmosfera, suelos, agua, brisa y neblina marina).

La atmosfera es un ambiente marino severo, por la distancia relativamente corta de $1.5 \mathrm{~km}$ con respecto al mar y presencia de brisa y neblina marina. El parámetro meteorológico que indica este resultado es la humedad relativa comprendida entre $60 \%$ a $98 \%$. Generalmente, este tipo de ambientes emiten cantidades $\geq 0,3 \%$ de $\mathrm{CO}_{2}$ y $>500 \mathrm{mg} / \mathrm{l}$ de $\mathrm{Cl}^{-}$, lo que lo hace un ambiente agresivo para los elementos de concreto (Sanchez de Guzman, 2004).

El suelo tiene valores de sales solubles de 7600 ppm, cloruros de 900 ppm y sulfatos de 2700 ppm, donde las cantidades de sales solubles y cloruros están dentro de los parámetros de la normativa E0.60 Concreto Armado; sin embargo, la cantidad de sulfato es muy severo que ocasiona el ataque químico a las cimentaciones.

El agua para la elaboración de concreto, curado y regadías de los sectores de áreas verdes son provenientes del Sector del Canal de Captación de Ite. Los valores de sulfatos de 600 ppm, cloruros de 240 ppm y pH de 8.71, donde las cantidades de cloruros y sulfatos están en los límites de los 
parámetros de la normativa NTP 339.088 Calidad de agua para el concreto; sin embargo, el valor de $\mathrm{pH}$ es relativamente excesivo. Las propiedades fisicoquímicas del agua

La brisa y neblina marina transportan importantes contenidos de humedad y consigo la presencia de cloruros y sulfatos. Según fundamentos teóricos e investigaciones, la composición química de la brisa del mar peruano comprende un contenido de cloruros de 23000 ppm y sulfatos de 42750 ppm y la composición química de la neblina es determinada por la atmósfera y la contaminación comprendiendo valores promedio de contenido de cloruros de $4.5 \mathrm{ppm}$ y sulfatos $<5$ ppm. Estos compuestos químicos son transportados por la dirección predominante del viento ocasionando que se depositen en la superficie del concreto donde inicia el mecanismo de daño (Bernal, 2009).

\section{Inspección detallada}

Los ensayos de laboratorio determinaron las propiedades mecánicas, físicas y químicas de los elementos de concreto y la aplicación de los estándares de la normativa evaluaron el estado de estas propiedades, para determinar el diagnostico.

La resistencia a comprensión mediante diamantinas de concreto, según la norma E.060 Concreto Armado establece que una buena calidad de concreto se considera si el promedio de los tres núcleos de concreto es $\geq 85 \%$ de $f^{\prime} c$ y que ningún núcleo tenga una resistencia $<75 \%$ de $f^{\prime} c$. Las vigas y columnas con fisuras leves tienen una resistencia admisible $\geq 75 \%$ de $f^{\prime} c$ cumpliendo con los estándares de la normativa. Sin embargo, Las vigas y columnas con fisuras moderadas y severas tienen una resistencia baja $<75 \%$ de $f^{\prime} c$ no cumpliendo con los estándares de la normativa. La baja resistencia de estos elementos es un índice de ataque químico inducidas por la presencia de cloruros que pueden reducir la resistencia de un $8 \%$ a $10 \%$ y $1 \%$ de sulfatos puede reducir la resistencia de un $10 \%$.

La absorción, densidad y porosidad del concreto, según el $\mathrm{ACl}$ establece que la densidad del concreto tiene un valor referente entre $2200 \mathrm{~kg} / \mathrm{m} 3-2400 \mathrm{~kg} / \mathrm{m}^{3}$ dependiendo de la cantidad y densidad de los agregados y la porosidad de un buen concreto tiene un límite de $10 \%$ en zonas urbanas (Diaz et al., 2002). La absorción del concreto tiene un valor promedio de 4,26 \%, lo que indica una característica de un buen concreto. La densidad aparente del concreto tiene un valor de 2 $584,11 \mathrm{~kg} / \mathrm{m}^{3}$, lo cual es referente a una reducción de la cantidad de pasta y un aumento de la cantidad de agregados; es decir, la densidad del concreto aumenta y genera una mayor resistencia del concreto. La porosidad del concreto tiene un valor promedio de $9,87 \%$, lo cual es un indicador de un buen concreto, pero permeable y no adecuado para ambientes agresivos marinos o cercanos al mar debido a la concentración de cloruros y sulfatos.

La profundidad de carbonatación se representa mediante la aplicación de fenolftaleína considerando el indicador de un concreto carbonatado que adquiere un color gris, mientras que un concreto no carbonatado ( $\mathrm{pH}$ mayor de 9) adquiere un color magenta. Para la parte exterior de las diamantinas de concreto la profundidad de carbonatación es de $12 \mathrm{~mm}$ que corresponde al recubrimiento. Para la parte interior de las diamantinas de concreto la profundidad de carbonatación es de 0,4 mm que corresponde al área del elemento de concreto. En este caso, el frente de carbonatación está presente en el recubrimiento y la presencia de carbonatación en el 
interior del elemento es mínima, lo cual no presenta peligro alguno para la capa de óxido pasivo que protege al acero de refuerzo de la corrosión.

El pH en el concreto debe tener un rango de 12 - 13 según la Norma $\mathrm{ACl}$, lo que garantiza un ambiente alcalino y característica de un buen concreto; y cuando el concreto esta carbonatado hacia el interior del acero de refuerzo tiene un $\mathrm{pH}<9,5$, lo que posibilita el inicio de corrosión. De las muestras de concreto se determina un valor de $\mathrm{pH}$ dentro de los estándares de la normativa; lo que indica que el concreto posee un pH estable y la perdida de alcalinidad es mínima en el concreto, es decir la capa de óxido pasivo que cubre el acero de refuerzo permanece estable (Rivva, 2014).

La cantidad de cloruros en el concreto, según la norma E.060 Concreto Armado establece el contenido máximo total de ion de cloruro proveniente de los ingredientes individuales del concreto y no aquellos provenientes del medio ambiente que lo rodea (Rivva, 2014). Las muestras de diamantinas de concreto representaron un porcentaje de ion de cloruro excesivo $(0,71 ; 0,83$ y 0,71 \%) fuera de los estándares de la normativa para concreto armado en exposición de acción de cloruros que establece un $0,15 \%$. En este caso la presencia de alto contenido total de cloruros en el concreto es proveniente de la contaminación de los ingredientes del concreto, lo que indica un posible daño por origen fisicoquímico, es decir, por corrosión del acero de refuerzo.

La cantidad de sulfatos en el concreto, según la norma E.060 Concreto Armado establece el contenido de sulfatos según el tipo de exposición, en la cual el Complejo Recreacional se encuentra en un ambiente agresivo moderado expuesto a sulfatos donde se establece un límite de 150 a 1500 ppm. Las muestras de diamantina de concreto representaron valores ( 1088,$5 ; 985,55$ y 1 409,20 ppm) dentro de los estándares de la normativa, lo que indica que la existencia de patologías en vigas, columnas y placas no es originada por la existencia de sulfatos en el concreto. En este caso, estas patologías no son originas por la expansión de la pasta producida por la reacción química de sulfatos.

La cantidad de carbonatación en el concreto, no se limita según el ACl; sin embargo, este dato es importante para evaluar el estado actual de las propiedades químicas del concreto. La muestra de diamantina de concreto de una columna con fisuras leves tiene un porcentaje mínimo de carbonatación. La muestra de diamantina de concreto de unas dos vigas con fisuras moderadas y grietas leves tiene un porcentaje medio y mínimo de carbonatación $(2,18 ; 13,55$ y $6,68 \%$ ). En este caso, la presencia de patologías en el concreto ha permitido el ingreso de $\mathrm{CO}_{2}$ a través de los factores como la humedad relativa, porosidad y recubrimiento dando origen a la carbonatación en los elementos de concreto (Montejo et al., 2012). En la Tabla 15, se observa la comparación de los resultados de carbonatación y pH en el concreto.

Por otro lado, los resultados obtenidos de los ensayos de laboratorio del concreto (figura 4) evidencian un incremento en las características fisicoquímicas del concreto entre el intervalo del periodo del 2016 al 2019, incremento de cloruros, incremento de sulfatos, disminución de pH y aumento de carbonatación, lo que explica la presencia de fisuras por corrosión en el acero de refuerzo en los elementos de concreto armado (vigas, columnas y placas). Efectivamente, no haber realizado una adecuada reparación en el año 2016 ocasionó que con el pasar del tiempo estos problemas patológicos se manifiesten nuevamente y se desarrolle mucho más el deterioro del concreto. 
Entre tanto, las características químicas de los agregados, según la norma NTP 400.042 establece el contenido máximo de cloruros y sulfatos en los agregados, lo cual comprende para el agregado fino un límite máximo en cloruros $<0,1 \%$ y un límite máximo en sulfatos $<1,2 \%$; y para el agregado grueso un límite máximo en cloruros $<0,1 \%$ y un límite máximo en sulfatos $<1,0 \%$. El agregado fino para la elaboración del concreto tiene un porcentaje de cloruros excesivo $(0,275$; 0,361 y $0,308 \%$ ), un porcentaje de sulfatos mínimos $(0,033 ; 0,026$ y 0,068$)$ cumpliendo los parámetros de la normativa. Asimismo, el agregado grueso para la elaboración del concreto tiene un porcentaje de cloruros y sulfatos mínimo de acuerdo a los parámetros de la normativa. En este caso, el ingrediente contaminado es el agregado fino debido a su alto índice de cloruros, factor que activa la corrosión del acero de refuerzo y por ende ocasiona la presencia de fisuras y grietas en vigas, columnas y placas. Los materiales contaminados son provenientes de las canteras cercanas al litoral, donde el viento y la brisa marina son factores que transportan cloruros, contenido químico natural de un ambiente marino.

La norma NTP 339.088 establece las características fisicoquímicas del agua para concreto, respecto a los límites máximos de $\mathrm{pH}$, cloruros y sulfatos en el agua para el concreto. El límite máximo de $\mathrm{pH}$ es de 5 a 8 , un límite máximo de cloruros de 1000 ppm y un límite máximo de sulfatos de 600 ppm. El agua del sector de captación de Ite, utilizado para la elaboración del concreto, tiene un $\mathrm{pH}$ de 8,71 que es excesivo según los estándares de la normativa, por el contrario los cloruros con 240 ppm y sulfatos máximos con 600 ppm, son valores que cumplen los estándares de la normativa. Los valores en cuanto al cumplimiento de la normativa NTP, se asemejan a la NORMA ASTM C-70 Agua de mezclado y curado. En este caso, la calidad del agua cumple con los estándares de calidad para el concreto y se descarta de ser ingrediente contaminante.

\section{Evaluación de patologías}

El nivel de severidad de los elementos con patologías predominante es de leves, moderados y severos; es decir, las estructuras se encuentran en un estado regular. En la Figura 5, se observa el nivel de severidad de los elementos de concreto de los diferentes ambientes del Complejo Recreacional. Las vigas, columnas y placas presentan fisuras y grietas en dirección del acero de refuerzo se presentan debido a un alto contenido de cloruros y carbonatación en el concreto proveniente de una atmósfera marina. Los sobrecimientos presentan eflorescencia debido a la falta de un sistema de drenaje y el alto contenido de cloruros y sulfatos del suelo lo que hace que el agua, proveniente del regadío de áreas verde, reactive las sustancias agresivas del suelo y se propague rápidamente a las cimentaciones. Las losas presentan humedad debido a la escasez de desfogue de agua lo que está sujeta a daños de corrosión en el acero de refuerzo. La escalera presenta fisuras y grietas debido a los deficientes procesos constructivos y al alto contenido de cloruros y carbonatación que generan la corrosión del acero de refuerzo.

\section{Diagnostico General}

El Complejo Recreacional está expuesto a la agresividad de un ambiente marino severo debido a la cercanía al mar y presencia de brisa y neblina marina; con características de alta humedad y concentración de cloruros y sulfatos. Las estructuras están cimentadas sobre suelo con elevado contenido de sales, cloruros y sulfatos; sin embargo, ante la presencia de humedad este sufre 
asentamientos debido a los cambios volumétricos que ocasionan la presencia de patologías en muros.

En referencia a las propiedades mecánicas, físicas y químicas del concreto, la resistencia del concreto en vigas, columnas y placas es relativamente baja del $f^{\prime} c$ de diseño en comparación al estudio que se realizó en el año 2016 y con respecto a la norma E0.60, es decir que el ataque químico ha reducido la resistencia del concreto. La existencia de ataque químico por cloruros y carbonatación posibilita el ingreso de agentes nocivos en contacto al concreto, por ello, se recomienda una resistencia de $f^{\prime} c=280 \mathrm{~kg} / \mathrm{cm} 2$, para elementos expuestos a ambientes agresivos con cercanía al mar.

La densidad del concreto es indicador de buen concreto con mayor cantidad de agregado grueso lo que representó una buena resistencia del concreto en el estudio realizado en el año 2016. La absorción del concreto tiene un valor promedio de 4,26 \% y la porosidad del concreto tiene un valor promedio de $9,87 \%$; lo que es indicador de un concreto poco permeable, pero no adecuado para ambientes agresivos.

La carbonatación se presenta en el área del recubrimiento y una mínima presencia en el área del mismo elemento de concreto; lo que da a entender la existencia de un frente de carbonatación mínima. El pH en el concreto tiene un valor promedio de 12.10, lo que señala la conservación de la alcalinidad del concreto y la estabilidad de la capa de óxido pasivo que cubre el acero de refuerzo. Sin embargo, el porcentaje de carbonatación en el concreto comprende valores de $2.18 \%, 6.68 \%$ y $13.55 \%$, razón que hace suponer que a pesar que el valor del pH del concreto se encuentre dentro de los estándares de la normativa, no precisamente indica la inexistencia de carbonatación.

El contenido de cloruros en el concreto tiene un valor promedio de 3932,15 ppm, y según la norma E0.60, no cumple con el límite permisible. Esto es un indicador de un ataque fisicoquímico y la razón del origen de fisuras y grietas en vigas, columnas y placas frente a la exposición en ambiente marino severo.

El contenido de sulfatos en el concreto tiene un valor promedio de 1,161.08 ppm y según la norma E0.60, cumple con el límite permisible. Esto indica que las patologías no son originas por la expansión de la pasta producida por la reacción química de sulfatos.

En referencia a las propiedades fisicoquímicas de los materiales utilizados para la elaboración del concreto se tiene el siguiente diagnóstico:

El agregado fino presento un porcentaje de cloruros excesivo y un porcentaje de sulfatos mínimos según los límites permisibles de la normativa. El agregado grueso presento un porcentaje de cloruros y sulfatos mínimos según los límites permisibles de la normativa; es decir, el ingrediente contaminado es el agregado fino debido a su alto índice de cloruros, factor que activa la corrosión del acero de refuerzo ocasiona fisuras y grietas en vigas, columnas y placas.

El agua utilizada en la elaboración el concreto proveniente del sector de captación de Ite es de buena calidad cumpliendo los límites permisibles de la calidad para el concreto y se descarta de ser ingrediente contaminante.

Debido a los resultados anteriores, se afirma que el origen de fisuras y grietas en vigas, columnas y placas se da por la presencia de cantidades considerables de cloruros en el concreto y 
por la exposición a un ambiente marino severo con alta humedad y concentración de cloruros y sulfatos.

Además, tras el examen general de patologías se identificó un patrón de fisuras similares en dirección al acero de refuerzo, lo cual conduce a problemas de corrosión. Las vigas, columnas y placas de los ambientes del Complejo Recreacional están diseñados por varillas de acero de 3/4", con un diámetro de $2 \mathrm{~cm}$ aproximadamente; que al presentar problemas de corrosión genera un incremento de volumen en el acero de refuerzo lo que ocasiona tensiones de tracción en el concreto, que al no poder ser soportadas por este produce fisuración en el mismo sentido de la barra de refuerzo.

Se puede añadir que, según antecedentes, en el año 2016 se realizó un mantenimiento en el Complejo Recreacional; el cual comprendió el resane de fisuras y grietas en vigas, columnas y placas y pintado de elementos de concreto. Sin embargo, no se realizó la reparación indicada a los elementos de concreto armado y como consecuencia nuevamente se presenciaron estos problemas patológicos, lo que origino que el ataque químico avance e incremente el contenido de cloruros y carbonatación.

En cuanto a fisuras en los muros de albañilería, se presentaron por razones de asentamiento del terreno ante la pérdida de consistencia mecánica ocurrida por la presencia de agua y sales solubles en el terreno de fundación; lo que genera vulnerables variaciones volumétricas en el suelo. Así mismo, la humedad y eflorescencia en sobrecimientos es producida por la acumulación de agua de regadío de áreas verdes y el contenido de sulfatos en el suelo, lo que ocasiona un ataque químico al concreto de las cimentaciones y este pueda ascender a los sobrecimientos.

$Y$ finalmente, con respecto a los problemas patológicos como el descascaramiento del concreto en columnetas y veredas, se relaciona al ataque físico por desgaste superficial - erosión debido a que las estructuras están expuestas a una atmósfera marina severa que contienen concentraciones de cloruros y sulfatos que, por medio del viento, se depositan en la superficie del concreto donde e inicia el mecanismo de daño (calavera, 2005).

\section{Conclusiones}

Tras la aplicación de los métodos de inspección preliminar, se concluye que el origen de los problemas patológicos en los elementos de concreto del Complejo Recreacional "Los Humedales de Ite" es consecuencia de no haber considerado el efecto de la exposición frente a un ambiente marino severo y la agresividad del contenido químico en el suelo en la etapa de inicio y planificación, lo que ha comprometido la durabilidad de los elementos de concreto.

Asimismo se concluye que la calidad del concreto es deficiente para una atmósfera marina severa debido a que el transporte de agentes nocivos se da través de la porosidad y absorción del concreto. Las características fisicoquímicas de los materiales indicaron que el ingrediente contaminado es el agregado fino debido a su alto índice de cloruros, factor que suma el proceso de corrosión del acero de refuerzo.

El Complejo Recreacional presenta patologías como: fisuras, grietas, eflorescencia, humedad y descascaramiento. El nivel de severidad de mayor incidencia es el nivel leve y moderado, donde las 
vigas, losas y sobrecimientos son los elementos de concreto con mayor número de patologías. El origen de fisuras y grietas en vigas, columnas y placas se da por la presencia de cantidades consideras de cloruros en el concreto. Estas fisuras tienen un patrón en dirección al acero de refuerzo, lo cual confirma problemas de corrosión. El ancho de las fisuras se da por el aumento de la tensión de las armaduras en relación al diámetro de las barras de acero de refuerzo.

\section{Referencias Bibliográficas}

ACI 201.2R. (s.f.). Guía para la durabilidad del hormigón. Lima: Comité ACl 201.

ACI 224.1R. (1993). Causas, evaluación y Reparación de fisuras en estructuras de hormigón.

ACl 562. (2016). Evaluación, reparación y rehabilitación de estructuras de hormigón. Lima: ACI.

DE CONCRETO, Asociación Colombiana de Productores. ASOCRETO. (2010). Tecnología y Propiedades.

ASTM-C1202, Standard Test Method for Electrical Indication of Concrete's Ability to Resist Chloride Ion Penetration. 2005.

Bernal, J. (2009). Durabilidad en estructuras de concreto armado, localizadas frente a la costa. México: Universidad Nacional Autónoma de México.

Calavera Ruiz, J. (2005). Patología de estructuras de hormigón armado y pretensado. España: INTEMAC.

Garzón Guzmán, J. M., \& Landin Romero, M. A. (2017). Manual de técnicas para rehabilitación y reforzamiento en columnas y vigas de hormigón armado deterioradas por agentes mecánicos. Ecuador.

Genesca, J. (1995). Corrosión y medio ambiente. México: Fondo de la Cultura Económica S.A.

Helene, P. (1983). Agresividad del medio y la durabilidad del hormigón. Buenos Aires.

Montejo Fonseca, A., Montejo Piratova, F., \& Montejo Piratova, A. (2013). Tecnología y patología del concreto armado. Bogotá: Universidad Católica de Colombia.

NTP E.060. (2009). Concreto Armado. Lima: SENCICO.

Pancorbo Floristán, F. (2013). Corrosión, degradación y envejecimiento de los materiales empleados en la edificación. México: Marcombo.

Porto Quintián, J. (2005). Patologías en las estructuras de hormigón armado. España.

Rivva López, E. (2014). Ataques al Concreto. Lima: ICG.

Sánchez de Guzmán, D. (2004). Durabilidad y Patologías del concreto. Lima: ASOCRETO. 